\title{
Automated 3D microphysiometry facilitates high-content and highly reproducible oxygen measurements within 3D cell culture models
}

\author{
Sebastian Eggert ${ }^{1,2,3, *}$, Martin S Gutbrod ${ }^{4}$, Gregor Liebsch ${ }^{4}$, Robert Meier ${ }^{4}$, Christoph \\ Meinert ${ }^{1,2}$, Dietmar W Hutmacher ${ }^{1,2,5, *}$ \\ ${ }^{1}$ Centre in Regenerative Medicine, Queensland University of Technology, Brisbane, 4000, QLD, Australia. ${ }^{2}$ \\ School of Mechanical, Medical and Process Engineering, Science and Engineering Faculty, Queensland \\ University of Technology, Brisbane, 4000, QLD, Australia. ${ }^{3}$ Chair of Medical Materials and Implants \\ Department of Mechanical Engineering and Munich School of BioEngineering, Technical University of \\ Munich, Garching, 85748, Germany. ${ }^{4}$ PreSens Precision Sensing GmbH, Am Biopark 11, 93053 Regensburg, \\ Germany. ${ }^{5}$ ARC ITTC in Additive Biomanufacturing, Queensland University of Technology, Brisbane, 4000, \\ QLD, Australia. \\ *Corresponding authors: diemtar.hutmacher@qut.edu.au, sebastian.eggert@tum.de.
}

Methods and Results

Evaluation of the positioning accuracy and repeatability

Characterization of temperature and oxygen dynamics

Figure S1. Concept visualization.

Figure S2. Characterization of the performance characteristics.

Figure S3. Application of the microphysiometry module for 2D monolayers.

Figure S4. Position of the temperature sensors.

Figure S5. Visualization of potential height differences due to meniscus shape.

Movie S1. Visualization of the measurement workflow.

Movie S2. Visualization of the microprofiling technique. 


\section{Evaluation of the positioning accuracy and repeatability}

Positioning accuracy specifies the deviation between the position as defined in the protocol script and the actual position as quantified by a measurement device. Positioning repeatability was defined as the range of the positional deviations obtained by a series of unidirectional movement to a target position. The positioning accuracy and repeatability was evaluated according to a previously established protocol ${ }^{1}$. To assess the positioning accuracy and repeatability of the implemented leadscrew-driven linear stage, two experiments were conducted with the transport module. First, a script was written to move the positioning module repeatedly to the lid lifter module position $(x=2 \mathrm{~cm})$ and the sensor module position $(x=80 \mathrm{~cm})$. On each module position, an image of a test target (USAF 1951, Thorlabs, Inc., USA) was acquired by a Raspberry Pi Camera Board (V2, Raspberry Pi Foundation, Cambridge, UK) connected to a Raspberry Pi (Raspberry Pi 3 Model $\mathrm{B}+$ ). This movement pattern was repeated for 32 times upon zero-point calibration. Secondly, the accuracy and repeatability of the positioning module were evaluated for a defined sample position (well H12 of 96-well plate) under the sensor module upon zero-point calibration. Acquired tiff images were processed with the ImageJ (National Institutes of Health) plugin Register Virtual Stack Slices² which exports the calculated displacement data (in pixels) between the uploaded images in name.xIm-files (XML: Extensible Markup Language). Using the USAF 1951 test target with defined patterns, a conversion factor can be applied to concert pixel information into micrometres. Positioning accuracy, also referred to as the systematic error, was calculated by:

$$
\begin{aligned}
& \mathrm{e}_{\mathrm{s}}=\bar{x}-x_{\text {test }} \\
& \left.\mathrm{e}_{\mathrm{s}} \quad \text { systematic error (in } \mu \mathrm{m}\right) \\
& \bar{x} \quad \text { arithmetic mean of measured positional deviations (in } \mu \mathrm{m})
\end{aligned}
$$


$x_{\text {test }}$ targeted position (in $\mu \mathrm{m}$ )

Positioning repeatability, also referred to as random error, was calculated by:

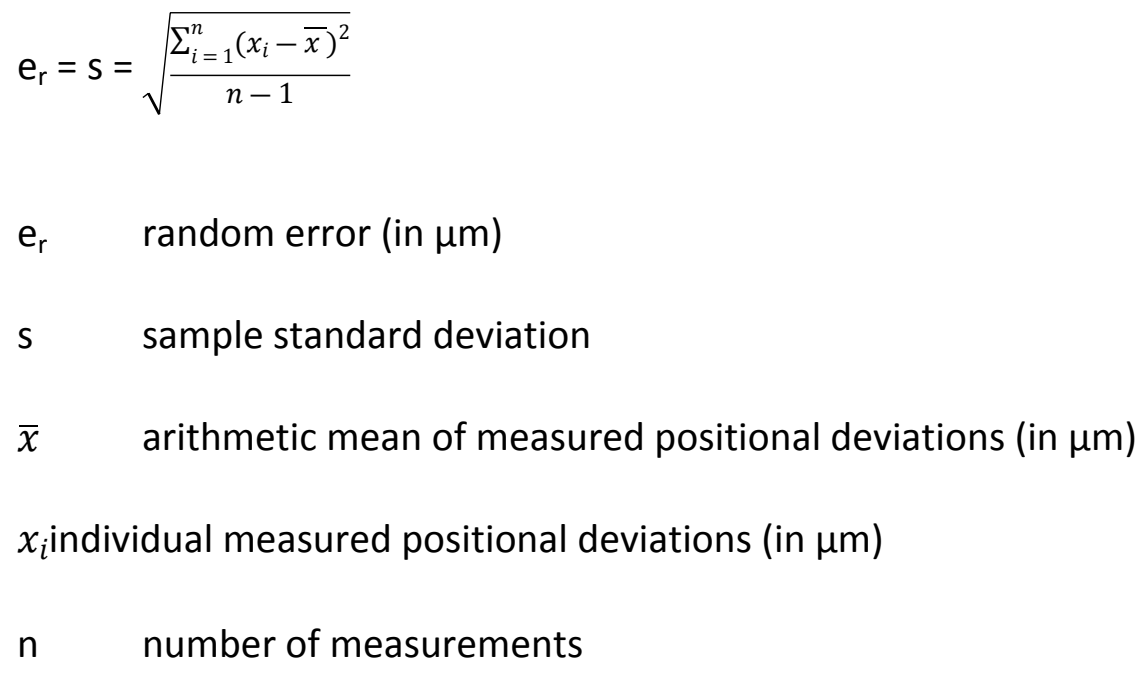

The realization of a fully automated and customized measurement workflow was enabled by the implementation of motorized positioning systems within the incubator. The operation of the motorized positioning system was validated and verified according to ISO 230-2:2014, which defines procedures for testing and evaluating the accuracy and repeatability of the positioning of numerically controlled axes ${ }^{3}$. According to ISO 230, accuracy describes the difference between the actual position recorded by a measurement device and the target position controlled by the operational unit, and repeatability expresses an estimator of the standard uncertainty of the positional deviations obtained by a series of measurements to a target position.

First, the positioning accuracy and repeatability of the transport module was assessed by unidirectional movements to the lid lifter unit $(x=2 \mathrm{~cm})$ and sensor unit position $(x=80 \mathrm{~cm})$ upon zero-point calibration (Figure S2/A-i). The deviation from the actual position to the target position is displayed in the graph in Figure S2/A-ii. From these data $(n=32$ per 
position), the mean and standard deviation were calculated for the lid lifter unit (81.7 $\mu \mathrm{m} \pm$ $5.1 \mu \mathrm{m})$ and sensor unit $(64.0 \mu \mathrm{m} \pm 28.5 \mu \mathrm{m})$ position. Next, the accuracy and repeatability of the positioning unit were evaluated for the most remote sample position (well H12 of 96-well plate: $55.7 \mathrm{~mm}$ in $\mathrm{x}$-direction and $100.9 \mathrm{~mm}$ in y-direction) under the sensor unit position upon zero-point calibration (Figure S2/A-iii). The minimal and maximal deviations from the target position vary between 2.3 and $165.3 \mu \mathrm{m}$ for $\mathrm{x}$-direction and -83.4 and $37.5 \mu \mathrm{m}$ for $\mathrm{y}$ direction, respectively (Figure S2/A-iv). The average deviation with the respective standard deviation were calculated for $x$ - (pink, $-14.7 \mu \mathrm{m} \pm 42.2 \mu \mathrm{m}$ ) and y-directions (green, $84.5 \mu \mathrm{m}$ $\pm 57.2 \mu \mathrm{m})$.

\section{Characterization of temperature and oxygen dynamics within the cell culture incubator}

Differences between manual sample positioning and an automated protocol were assessed by monitoring changes of the temperature and oxygen in the incubator. To compare the execution of an automated protocol with manual-based sample positioning from a previous microprofiling study ${ }^{4}$, we mimicked the manual-based plate positioning which took 30 seconds with an open cell incubator door (information about the time was received from authors ${ }^{4}$ ). In comparison to the manual positioning, an automated protocol was executed to identify general changes in temperature and oxygen within the incubator. Each experiment was started after waiting at least $24 \mathrm{~h}$ to reach temperature and oxygen equilibrium within the incubator. The continuous operation of stepper motors generates heat, as the control board supplies the stepper motors with the required current to drive the linear stage. The generated heat adds to additional heating of the incubator environment. To evaluate whether the incubator temperature maintains the culture temperature of $37^{\circ} \mathrm{C}$, the temperature changes over time were recorded during the execution of a measurement protocol at three different positions (sample position, motor position, environment position) (Figure S4). 
Temperature changes were measured with temperature probes (DS18b20, AusElectronicsDirect, Sydney, NSW, Australia) connected to a microcontroller board (Arduino Mega 2560, Arduino SRL, Italy).

Current microprofiling techniques require manual tasks ${ }^{4}$, such as incubator opening and closing, resulting in temperature and oxygen fluctuations and an increased risk for contaminations. A single manual position task of 30 seconds leads to an instant temperature drop of $2.3{ }^{\circ} \mathrm{C}$ and an oxygen increase of $1.2 \%$ at the sample location (Figure S2/B-i). While temperature and oxygen values recover to baseline values after 70 and $40 \mathrm{~min}$ for on-off manual positioning, respectively, repeated manual positioning tasks resulted in a wave-like decreasing temperature and increasing oxygen values over time (Figure S2/B-ii). In comparison to manual positioning tasks, the execution of an automated measurement protocol does not affect temperature and oxygen values (Figure S2/B-iii), and thereby provides a reproducible culture environment and decreased risks for sample contamination. This feature is particularly important for cells culture experiments performed at nonatmospheric oxygen concentrations, such as under hypoxia. Already a slight, temporary increase of the oxygen concentration may falsify the data.

Since the operation of the motorized positioning systems generates heat within the stepper motors which is emitted to the incubator environment, temperature changes were recorded (Figure S2/C-i,ii) during the execution of a measurement protocol with temperature probes at three different positions (Figure S4). While the temperature probe next to the stepper motor indicated an instant temperature increase after starting the protocol, probes in the middle of the incubator and next to the sample position exhibited an increase of $0.1^{\circ} \mathrm{C}$ within the first $30 \mathrm{~min}$. At the end of the measurement protocol (ca. $115 \mathrm{~min}$ ), the sample temperature increased to $37.2^{\circ} \mathrm{C}$. After protocol completion, the temperature dropped for 
all three positions and reached initial values after $10 \mathrm{~min}$ for the sample temperature and after $70 \mathrm{~min}$ for the motor temperature. These measurements of the temperature changes during operation highlighted that the generated heat of the stepper motors results in a notable temperature increase. However, the conducted temperature experiments demonstrated that the sample temperature is constant at $37{ }^{\circ} \mathrm{C}+/-0.3{ }^{\circ} \mathrm{C}$ degrees for a measurement protocol.

\section{References}

(1) Schneidereit, D.; Kraus, L.; Meier, J. C.; Friedrich, O.; Gilbert, D. F. Step-by-Step Guide to Building an Inexpensive 3D Printed Motorized Positioning Stage for Automated High-Content Screening Microscopy. Biosens. Bioelectron. 2017, 92 (August 2016), 472-481. https://doi.org/10.1016/j.bios.2016.10.078.

(2) Arganda-Carreras, I.; Sorzano, C. O. S.; Marabini, R.; Carazo, J. M.; Ortiz-de-Solorzano, C.; Kybic, J. Consistent and Elastic Registration of Histological Sections Using Vector-Spline Regularization. In In: International Workshop on Computer Vision Approaches to Medical Image Analysis. Springer (Berlin, Heidelberg).; 2006; pp 85-95. https://doi.org/10.1007/11889762_8.

(3) International Organization for Standardization. ISO 230-2:2014 - Test Code for Machine Tools - Part 2: Determination of Accuracy and Repeatability of Positioning of Numerically Controlled Axes, 2014.

(4) Wolff, P.; Heimann, L.; Liebsch, G.; Meier, R. J.; Gutbrod, M.; van Griensven, M.; Balmayor, E. R. OxygenDistribution within 3-D Collagen I Hydrogels for Bone Tissue Engineering. Mater. Sci. Eng. C 2019, 95 (1), 422-427. https://doi.org/10.1016/j.msec.2018.02.015. 

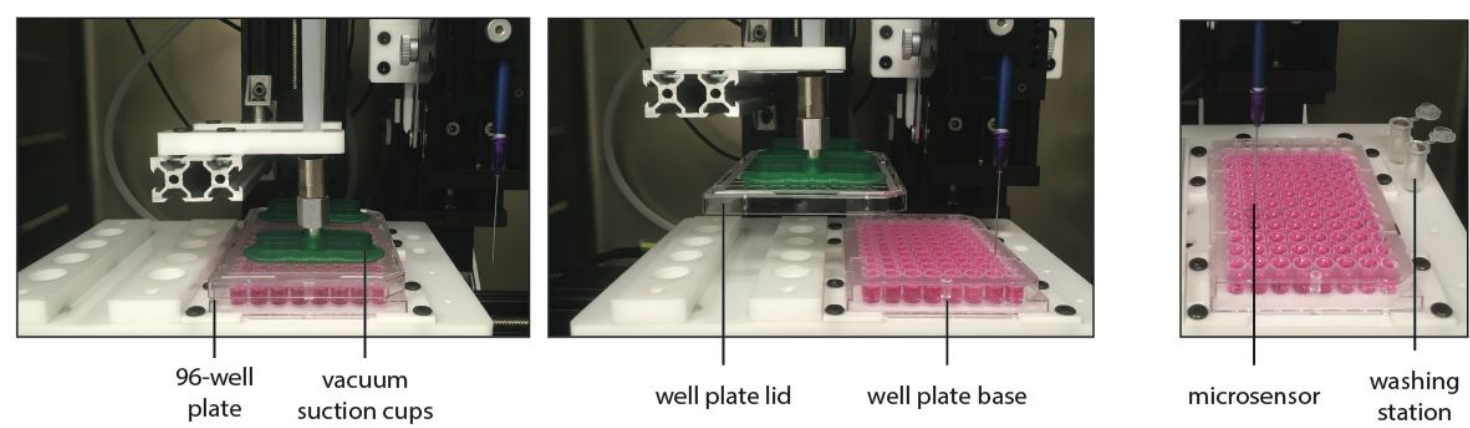

C
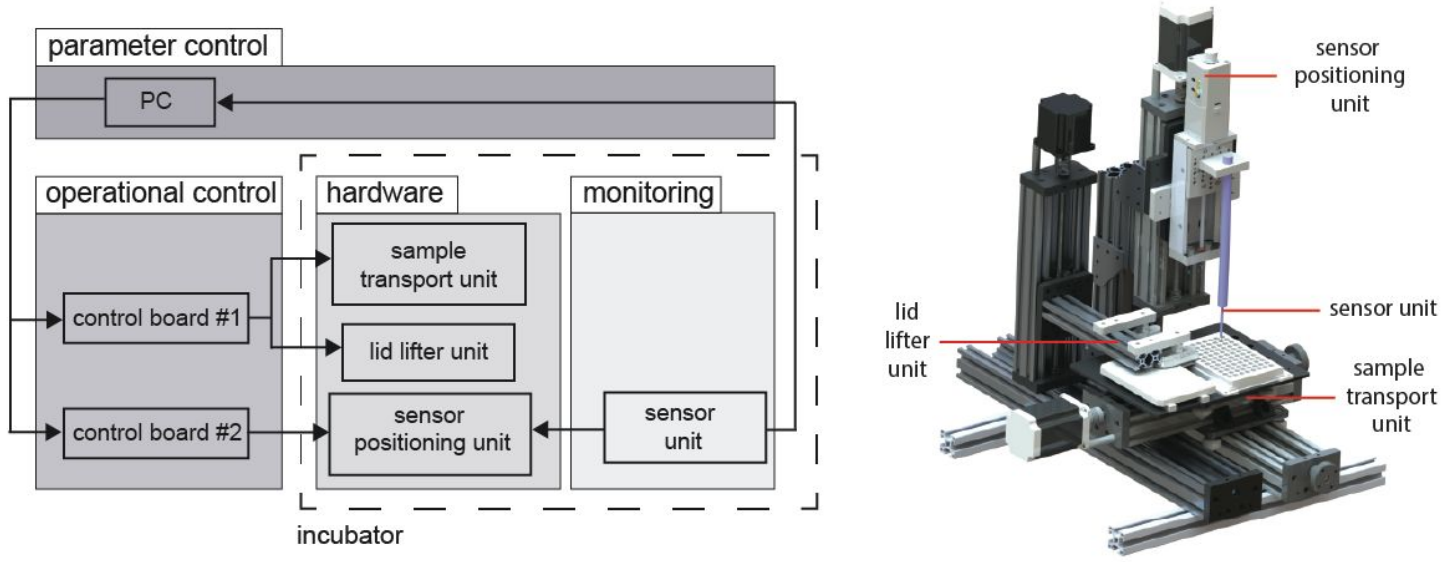

Figure S1. Concept visualization. (A) The photographs show the system installed in a cell culture incubator and a close-up of the sample table. (B) By automated opening and closing of the well plat lid, a fully automated workflow without human intervention is realized with the implemented system. A movie highlighting the operation of the setup is available (movie S1, movie S2). (C) The system architecture is shown in abstract layer, comprising parameter control, operational control, hardware, and monitoring. (D) The CAD rendering shows the designed workstation with the following functional hardware units: sensor positioning unit, lid lifter unit, sensor unit, and sample transport unit. 
(i) unit positioning

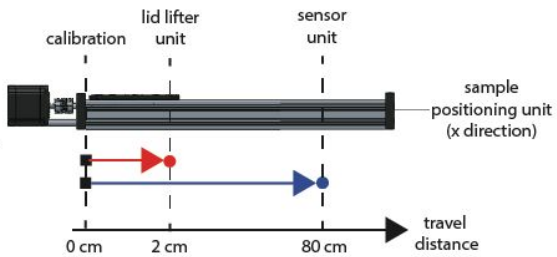

(ii) deviation from unit position

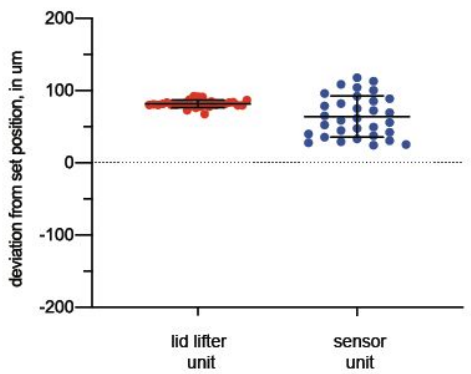

(iii) sample positioning unit

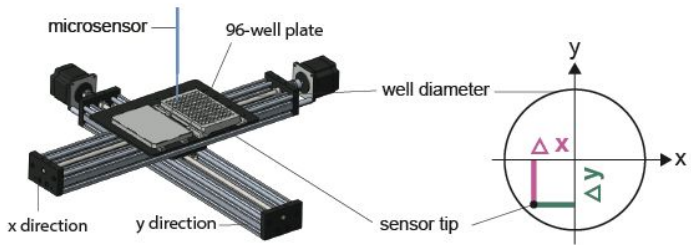

(iv) $\mathrm{x}$ and $\mathrm{y}$ deviation from sensor tip

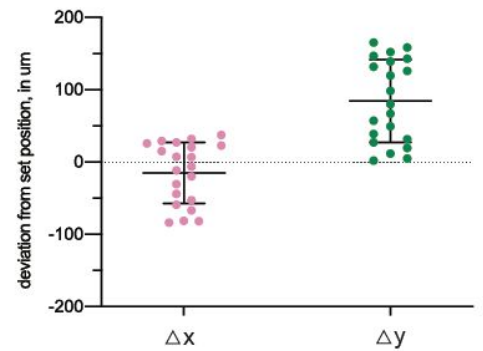

(i) on-off manual positioning
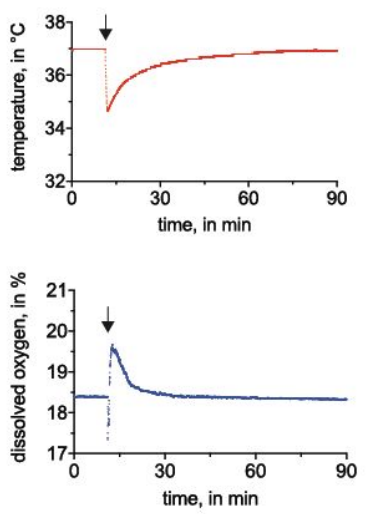

(ii) repeated manual positioning
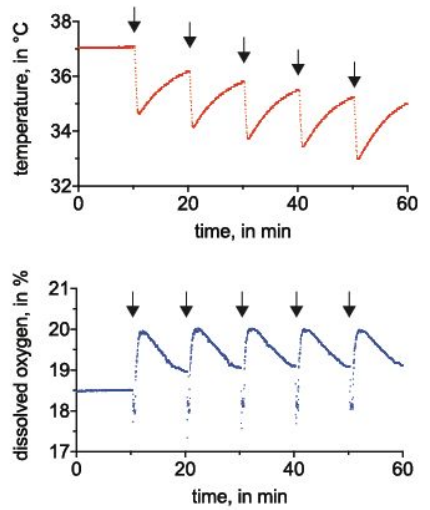

(iii) automated protocol
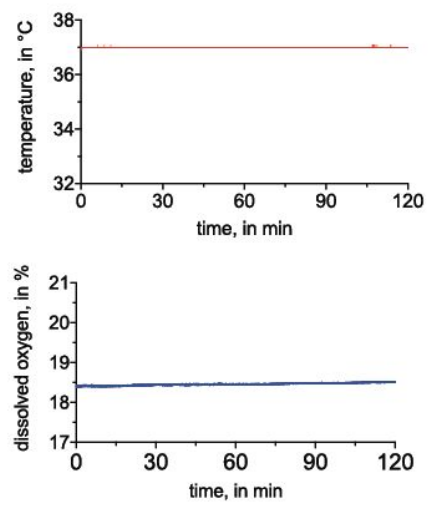

C temperature distribution within the incubator during protocol execution

(i)

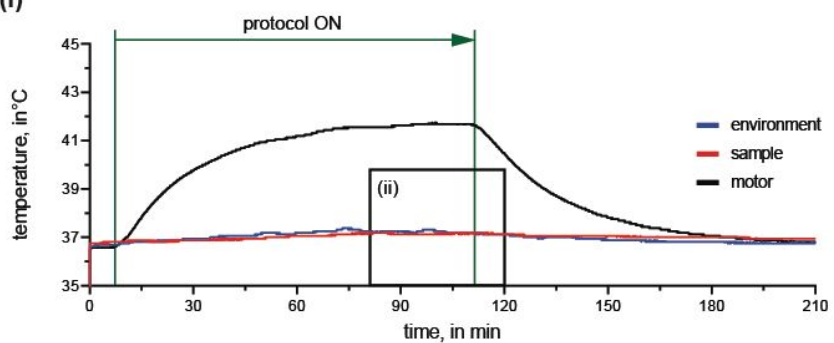

(ii)

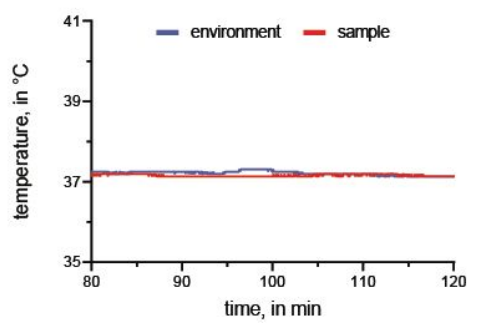

Figure S2. Characterization of the performance characteristics. (A) The accuracy and precision of the motorised positioning system is evaluated for (i, ii) the x-axis of the positioning unit by moving to the lid lifter unit and sensor unit, and for (iii, iv) the sample positioning unit. (B) The temperature (red) and oxygen (blue) dynamics were assessed between (i) on-off manual positioning, (ii) repeated manual positioning and (iii) an automated protocol. (C) The influence of the generated heat by the stepper motors is evaluated for (i) an entire protocol length and (ii) shown in more detail. 
A

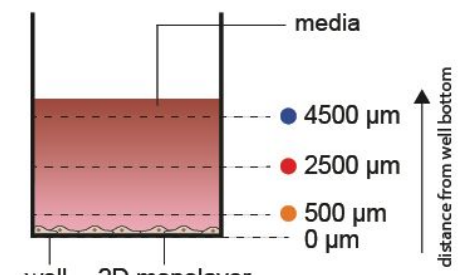

well 2D monolayer bottom

$$
\text { seeding density }
$$

- 5000 cells/well

- 10000 cells/well

20000 cells/wells

C day 2

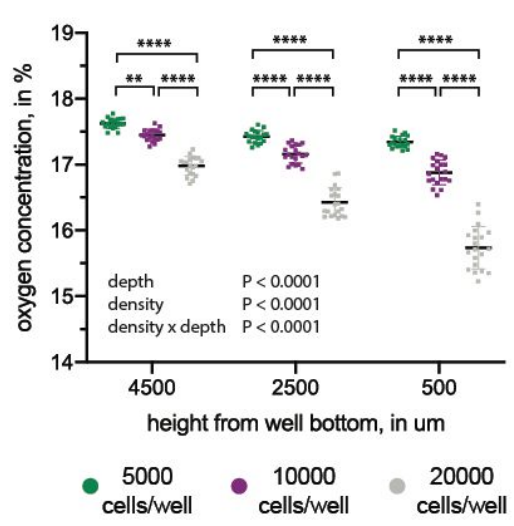

B oxygen tension at $500 \mu \mathrm{m}$

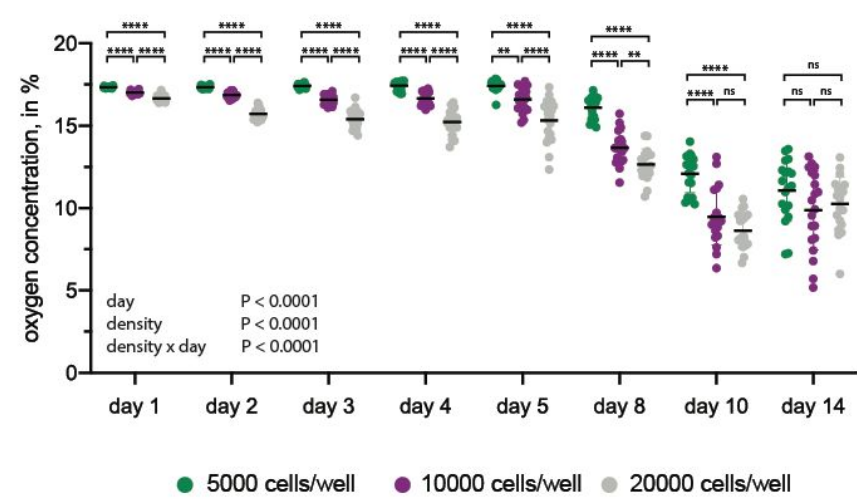

D seeding density: 20000 cells per well

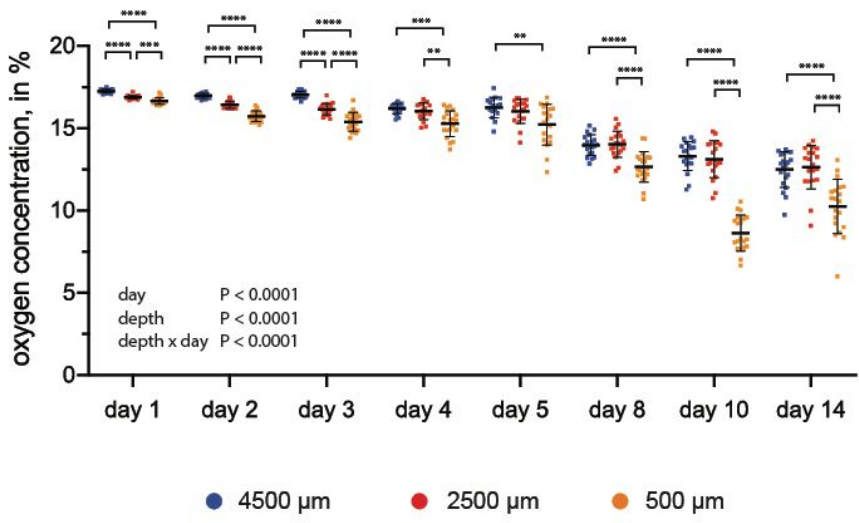

Figure S3. Application of the microphysiometry module for 2D monolayers. (A) Illustration of the culture set-up and the measurement schema. (B) Oxygen tension at a distance of 500 um from the well plate bottom for three cell seeding densities over 14 days. (C) Results of the oxygen measuremets for three different cell seeding densities on day 2. (D) Results of the oxygen measurements with the highest cell seeding density over 14 days. Means \pm standard deviation, $\mathrm{n}=20$ per cell seeding density, ${ }^{* * * *} \mathrm{P}<0.0001,{ }^{* * *} \mathrm{P}<0.001, * * \mathrm{P}<0.01, * \mathrm{P}<0.05$. 


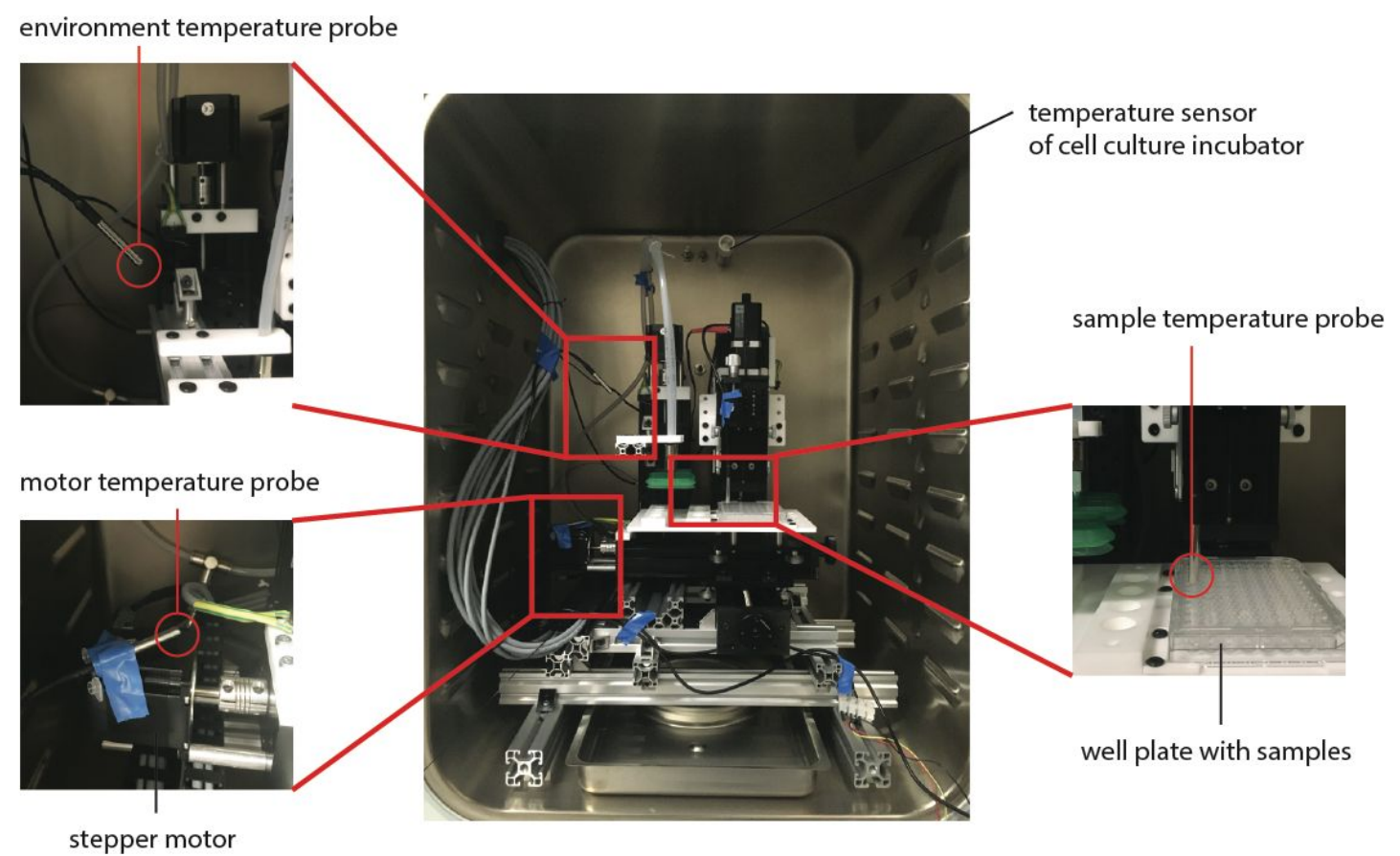

Figure S4. Position of the temperature sensors. 


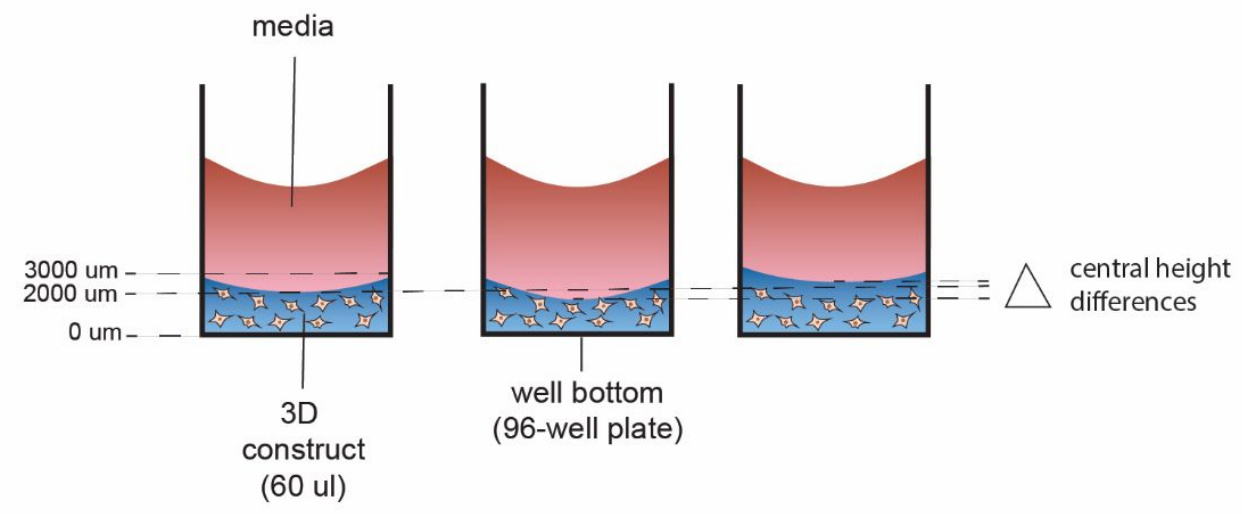

Figure S5. Visualization of potential height differences due to meniscus shape. 


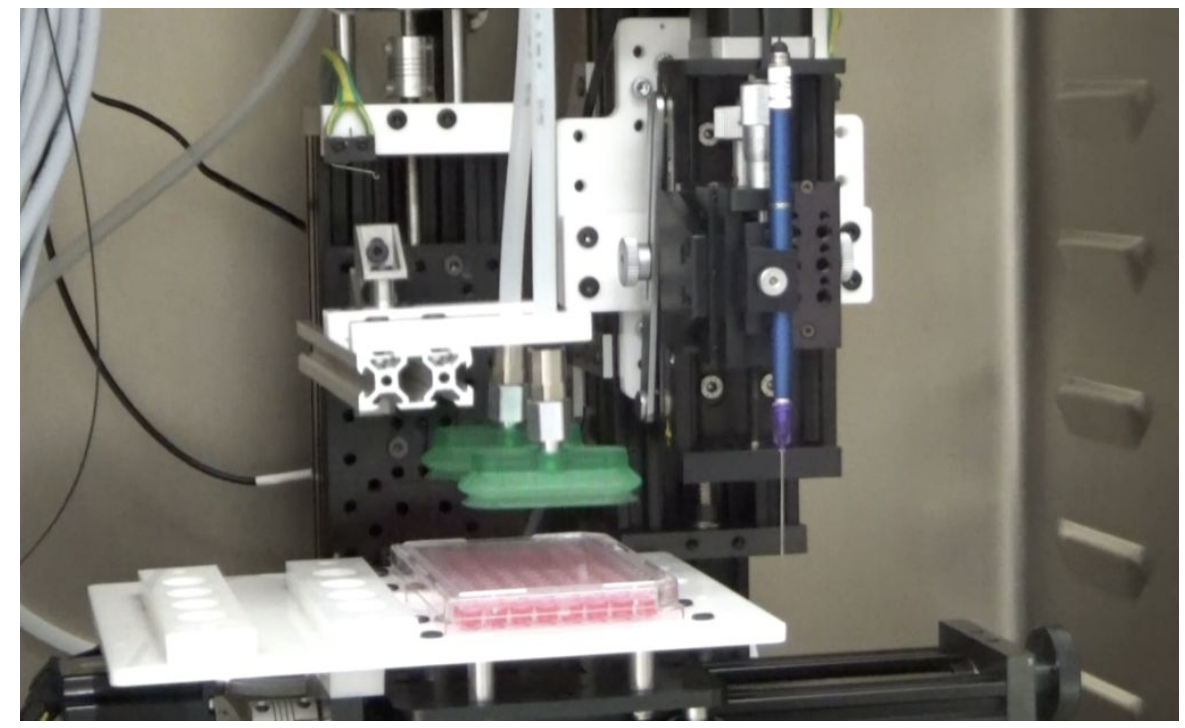

Movie S1. Visualization of the measurement workflow.

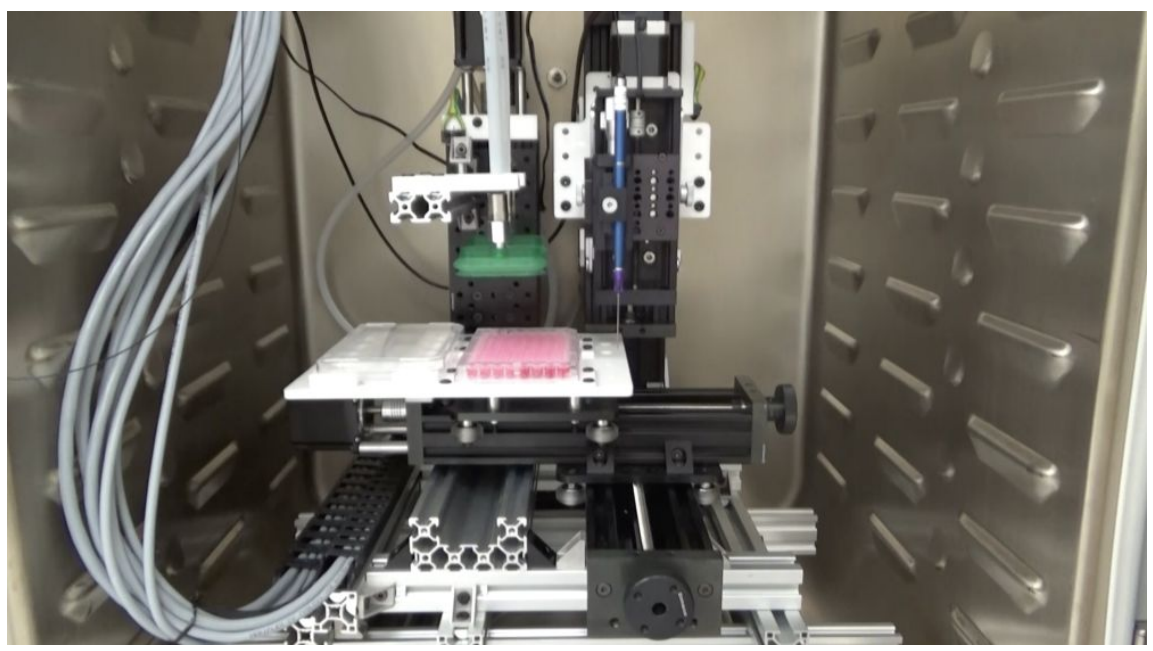

Movie S2. Visualization of the microprofiling technique. 